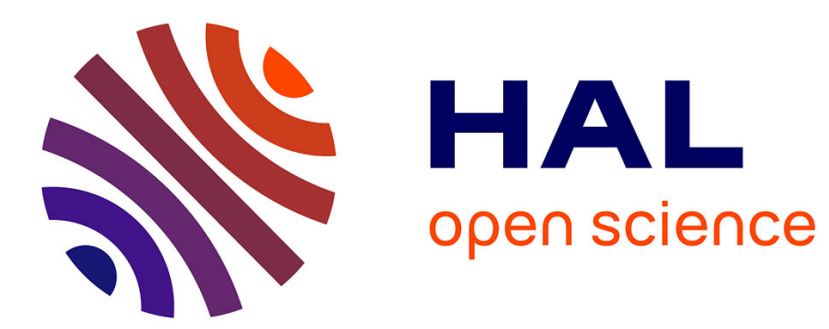

\title{
EFFECT OF THE HEAT BATH ON ACTIVATED RATE PROCESSES IN SOLIDS
}

\author{
R. Bauer
}

\section{To cite this version:}

R. Bauer. EFFECT OF THE HEAT BATH ON ACTIVATED RATE PROCESSES IN SOLIDS. Journal de Physique Colloques, 1985, 46 (C10), pp.C10-27-C10-30. 10.1051/jphyscol:19851005 . jpa00225293

\section{HAL Id: jpa-00225293 https://hal.science/jpa-00225293}

Submitted on 1 Jan 1985

HAL is a multi-disciplinary open access archive for the deposit and dissemination of scientific research documents, whether they are published or not. The documents may come from teaching and research institutions in France or abroad, or from public or private research centers.
L'archive ouverte pluridisciplinaire HAL, est destinée au dépôt et à la diffusion de documents scientifiques de niveau recherche, publiés ou non, émanant des établissements d'enseignement et de recherche français ou étrangers, des laboratoires publics ou privés. 
JOURNAL DE PHYSIQUE

Colloque $C 10$, supplément au $\mathrm{n}^{\circ} 12$, Tome 46 , décembre 1985 page $\mathrm{Cl0}-27$

\title{
EFFECT OF THE HEAT BATH ON ACTIVATED RATE PROCESSES IN SOLIDS
}

\author{
R. BAUER \\ Max-PIanck-Institut für Metallforschung, Institut für Physik, \\ Heisenbergstr. I, D-7000 Stuttgart 80 \\ und \\ Institut für Theoretische und Angewandte Physik der \\ Universitat stuttgart, Pfaffenwaldring 57 \\ D-7000 stuttgart 80, F.R.G.
}

Abstract - The effect of heat-bath coupling on the conditions of access to saddle-point configurations of vacancy jumps in solid-state diffusion is investigated by analysing, in the framework of quantum statistics, the interplay between i) the rate of phonon-phonon collisions caused by anharmonicity, representing the bath rate, $i i)$ the resonant mode of the defect and its 1 ifetime determined by the additional anharmonicity caused by the defect, and iii) the rate of barrier relaxation of the multivariate deformable barrier. At low temperature where damping is small, the resulting jump rate closely approaches the rate law of Vineyard (1957) characterized by the fully relaxed saddle point.

With increasing temperature, damping of the resonant mode increases, allowing but for a partial relaxation; jumps occur at reduced rate over nonequilibrium configurations of the saddle point with increased migration enthalpy and, caused by large spatial gradients of the energy, with enlarged values of migration entropy and activation volume. The typical non-Arrhenius form of the rate resulting from temperature-dependent damping allows to interpret self-diffusion in many metals by jumps of monovacancies only.

Anharmonic damping of the resonant mode involves essentially low-frequency phonons not contained in the spectrum of crystallites with up to 1000 atoms. Computer modelling of jump rates thus underestimates the relevant damping and yields always the low-temperature approximation, that is the Vineyard rate.

\section{I - INTRODUCTION}

The temperature dependence of experimental data of the tracer self-diffusion coefficient $D^{T}$ of metals usually exhibits curved Arrhenius plots $1 \mathrm{~g} \mathrm{D}^{\mathrm{T}}$ vs $1 / \mathrm{T}, / 1 /$; the curvature is strong for bcc metals like $\mathrm{Nb}, \mathrm{Ta}, \mathrm{Mo}, \mathrm{W}$ and $\mathrm{Na}$ while it is small but experimentally well established for the fcc metals $\mathrm{Cu}, \mathrm{Ag}, \mathrm{Au}, \mathrm{Ni}, \mathrm{Pt}, / 1-3 /$. This curvature may be caused either by the contributions $D_{i}^{T}$ of at least two migrating types of intrinsic atomic defects $i$ with suitably differing activation parameters, or by curvature of $D_{I}^{T}=f_{1} a^{2} c_{1} \Gamma_{1}$ of one defect only, due most likely to its jump rate $\Gamma_{1}$. The jump rate of atomic defects has so far been estimated by transition state theory (TST) $/ 4,5,6 /$ and its extensions /7,8/ based on the assumption of the equilibrium distribution of states and implying the relaxed vineyard sadile point $\mathrm{SP}_{\mathrm{Vin}}$. The activation enthalpy of this saddle point depends but little on temperature /9/; hence TST supports two-defect models of self diffusion /10,11,12/.

Any rate theory which is more complete than TST has to evaluate the deviations from the equilibrium distribution. This affords consideration of the coupling to the heat bath, which on the one hand establishes a temperature $\beta^{-1}=k_{B}{ }^{T}$ and theirmal fluctuations providing for hopping of atomic defects, and on the other hand imposes damping or dissipation on the dynamical motions. These two effects of a heat bath are inseparably interconnected like either side of one coin, as it is expressed in 
the fluctuation-dissipation theorem, of. /13/. TST neglects dissipation and establishes thereby an upper bound for any rate. Besides the early work of Kramers /14/ on this topic, several hundred papers have appeared in the past eight years, cf. /15/ for a review, dealing often with reactions in molecules immersed in a solvent. The present note discusses the elements to treat the jump rate of atomic defects in crystals for the example of the monovacancy in metals.

II - THE ONE-DIMENSIONAL PROBLEM

We consider a particle with mass $M$ in $a$ potential $U(x), F i g .1$, describing a metastable well at $x=0$ and a barrier with height $\mathrm{U}^{\mathrm{b}}$ at $\mathrm{x}^{\mathrm{b}}$. The curvatures in the bottom and at the barrier are measured by $\omega^{\circ}$ and $\omega^{\mathrm{b}}>0$, respectively. Coupling to a heat bath provides the inverse temperature $\beta$ and imposes a friction $\zeta(x)$ or a damping rate $\gamma(\mathrm{x})=\zeta(\mathrm{x}) / \mathrm{M}$. TST yields the rate of escape

$$
\Gamma_{\text {TST }}=\nu^{0} \exp \left(-B U^{b}\right)
$$

we use $\omega=2 \pi \nu$ throughout. Neither $\omega^{b}$ nor $\gamma$ are contained in this rate Iaw. Kramers 114/ found that in the limit of low friction (I) the thermal activation in the well is the rate-limiting process and the rate is proportional to $\gamma^{\circ} \approx \gamma(0)$ : with the transmission coefficient $r \equiv \Gamma / \Gamma_{\text {TST }}$, he obtained

$$
\mathbf{r}_{I}=\gamma^{\circ} \beta U^{b} / \nu^{\circ} \text { for } \gamma^{\circ} \rightarrow 0 \text {. }
$$

For intermediate and high friction (II), activation of the particle to the barrier is so fast that escape over the barrier is the rate-determining process. There, damping at the barrier $\gamma^{b} \approx \gamma\left(x^{b}\right)$ is essential in reducing the equilibrium rate $\Gamma_{\text {TST. }}$ With the normalized barrier damping $\alpha \equiv \gamma^{b} / 2 \omega b$ the result is

$$
r_{I I}=\omega_{R}^{b} / \omega^{b}=\alpha\left\{\left(1+1 / \alpha^{2}\right)^{I / 2}-1\right\}, \quad \alpha \geq 1 / 6,
$$

yielding $r_{I I} \rightarrow 1 / 2 \alpha=\omega^{b} / \gamma^{b}$ for $\alpha>1$ and $r_{I I} \approx 0.8$ for $\alpha=1 / 6$. $\omega_{R}^{b}$ and $\omega^{b}$ are the friction-induced and the bare transmission frequencies of the barrier, respectively. This expression, formerly often judged as an approximate interpolation formula, is now well established: it appears as the classical limit of the quantum-statistical rate for linear coupling to the bath and for ohmic or viscous damping $/ 16,17 /$, and it is obtained for non-Markovian dynamics /18,13/ where a memory-renormalized $\hat{\alpha}$ replaces $\alpha$.

The complete solution combining the regimes I and II for arbitrary but uniform damping $\gamma=$ const $(x)$ is worked out in $/ 13 /$, approximate solutions are given in $119,20 \%$. In regime $I$, $U^{b}$ in the exponent is replaced by $U_{1}(\gamma)$ with $U_{1} \rightarrow U^{b}$ for $\gamma \rightarrow 0$ and $\mathrm{U}_{1} \rightarrow 0$ if $r_{I}$ approaches unity /13/. This decrease of the activation energy in the transition region is a new result not obtained in the Kramers limits.

III - THE MULTT-DIMENSIONAL CASE

The rate constituting the diffusion coefficient of a monovacancy is the jump rate of one nearest neighbour of the vacancy over the barrier formed mainly by one gate of four gate atoms in the fcc lattice and by two consecutive gates of three atoms each in the bcc lattice. Various saddle-point configurations $S_{C}$ are distinguished by $c$; the equilibrium saddle point is SPVin. The infinitesimal potential at extreme points is described by mass weighted normal co-ordinates $q_{\lambda}, q_{\mu}$ and normal mode frequencies $\omega_{\lambda}, \omega_{\mu}$ where $\lambda$ and $\mu$ count modes in the ground state at the bottom of the well and at the top of the energy barrier, respectively:

$$
2 \mathrm{U}\left(\mathrm{q}_{\lambda}\right)=\Sigma_{1}^{\mathrm{N}} \omega_{\lambda}{ }^{2} \mathrm{q}_{\lambda}{ }^{2} ; \quad 2 \mathrm{U}\left(\mathrm{q}_{\mu}\right)=2 \mathrm{U}_{\mathrm{c}}-\left(\omega_{\mathrm{c}}^{\mathrm{b}}\right)^{2} \mathrm{q}_{1}{ }^{2}+\Sigma_{2}^{\mathrm{N}}\left(\omega_{\mu}^{\mathrm{c}}\right)^{2} \mathrm{q}_{\mu}{ }^{2} \text {. }
$$


Here $w_{c}^{b}$ is the bare transmission frequency and $N$ the total number of degrees of freedom. These harmonic modes are mutually coupled by the anharmonic terms of the lattice potential leading to the damping parameters $\gamma_{\lambda}, \gamma_{\mu} / 21 /$

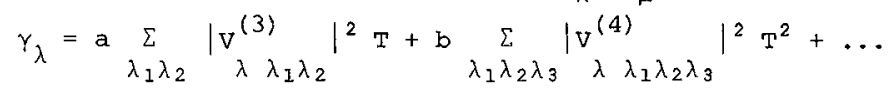

and a corresponding expression for $\gamma_{\mu}$. The $v_{\lambda}^{(n)}$... and $v_{\mu}^{(n)}$. depend on the $n$-th derivative of the potential about the well botton and the barrier top, respectively. For the stable modes the contributions to the $\gamma$ are interpreted as $n$-phonon collisions which for $T>T_{E}\left(=\right.$ Einsteintemperature) are $\sim T^{(n-2)}$. The $\gamma_{\lambda}$ are the phonon widths or the inverse of the lifetimes $\tau_{\lambda}$. The entirety of modes $\lambda$ or $\mu$ represent the heat bath coupled by the anharmonicities of the potential to the modes involved immediately in the dynamics of the jump process. In the perfect lattice, theory $/ 22 /$ and experiment /23/ indicate $\gamma_{\lambda} \leqslant 0.5 \omega_{\lambda}$ at $\mathrm{T}=\mathrm{T}_{\mathrm{m}}$ and $\gamma_{\lambda} \approx 0.05 \omega_{\lambda}$ for $\mathrm{T} \rightarrow 0$. The vacancy introduces strong additional anharmonicities, especially at the barrier, where on symmetry grounds the even coefficients are essential for the transmission damping: $\gamma_{C}^{b} \approx B_{C} T^{2}+D_{C} T^{4}$. The normalized barrier damping $a_{C}=\gamma_{C}^{b} / 2 \omega_{C}^{b}$ depends thus on the configuration $C$ and on $T$.

The TST result for the vacancy has been given by Vineyard /5/

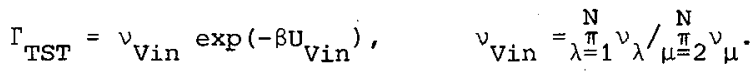

Again neither $\omega_{C}^{b}$ nor any damping parameter do appear in this expression.

The regime I of low damping $\gamma_{\lambda} \ll \omega_{\lambda}$ where vibrational energy transfer in the well is rate limiting is possibly realized for $\mathrm{T} \rightarrow 0$. Assuming that $\mathrm{N}_{\mathrm{C}}$ modes $\lambda$ have to be excited to reach $\mathrm{SP}_{\mathrm{C}}$, the reduced rate in $\mathrm{N}_{\mathrm{C}}$ dimensions is $/ 24,25 /$

$$
r_{I} \leqslant \gamma\left(\beta U_{C}\right)^{N_{C}} \exp \left(-\beta\left(U_{C}-U_{V i n}\right)\right) /\left(N_{C} ! v_{V i n}\right), \quad \gamma=\sum_{\lambda}^{N_{C}} \gamma_{\lambda}
$$

going to the kramexs limit for $N_{C}=1$. Since $\beta U_{C} \gg 1$ at low $T$, the rate is dramatically increased with respect to the one-dimensional case and the turnover between I and II /25/ should hardly be observable in solid-state diffusion; the TST rate is expected to hold at low $T$ until $\alpha_{c}$ starts to increase.

Regime II where barrier damping reduces successful barrier crossings is governed by $\alpha_{C} \cdot$ Expanding (3) we obtain

$$
r_{I I} \approx \omega_{C}^{b} \exp \left(-\beta\left(U_{c}-U_{V i n}\right)\right) / \gamma_{c}^{b}(T)
$$

which for fixed $c$ is an exponential whose pre-factor decreases as $\mathrm{T}^{-2}$ or $\mathrm{T}^{-4}$ with increasing temperature. The magnitude of the pre-factor depends on the ratio of the harmonic to the anharmonic parts of the barrier and varies with $\mathrm{c}$. Consider the bcc lattice with its double-gate structure of the barrier of the vacancy jump. In the relaxed state $c=$ Vin it tends to be close to a rectangular barrier with very small $\omega^{b}$ and large $\gamma^{b}$. This case exhibits a strong reduction of $r_{I I}$ by damping due to numerous recrossings of the diffusing particle before it moves away from the barrier. For the unrelaxed or partially relaxed barrier with $U_{C}>U_{V i n} \omega_{C}^{b}$ is increased and $\gamma_{C}^{b}$ decreased, and it depends on $\beta$ what configuration is the most favourable: at intermediate $T<T_{m} / 2$ the influence of the exponential is strong and $U_{C} \approx U_{V i n}$ with a decreasing pre-factor is preferable while at high temperature $T \approx T_{m}$ the decrease of the pre-factor would be severer and the largest contribution to the rate comes from jumps over non-equilibrium configurations of the partially relaxed barrier with increased migration enthalpy and, caused by the finite spatial gradients of $U_{C}$, with enlarged values of migration entropy and activation volume. These are the very features found in the analysis of self-diffusion in bcc metals; two-defect models $/ 10$ $-12 /$ to explain these properties are clearly not supported or postulated by the present rate theory which goes beyond TST by estimating all implications of the heat bath coupling. For fcc metals the effects are similar but less pronounced due to the simpler barrier structure. It appears that the recrossings due to damping are at least as important as the correction caused by dynamical return jumps /7,8/ associa- 
ted with anharmonicity and curvature of the saddle surface.

ACKNOWLEDGEMENTS. The author would like to thank $\mathrm{H}$. Schultz for continuous critical discussion during one year. He appreciates comments by D. Lazarus, C.P. Flynn, G. Delorenzi, A.V. Granato; R. Benedek, J.N. Mundy, N.L. Peterson and R.w. Siegel; H. Grabert and $U$. Weiss. The work is supported by the Bundesminister für Forschung und Technologie.

\section{REFERENCES}

/1/ Siegel, R.W., in Yamada Conference V on "Point Defects and Defect Interaction in Metals", University of Tokyo Press 1982, p. 533.

/2/ Peterson, N.I., J. Nucl. Mat. 69 \& 70 (1978) 3.

13/ Mehrer, H., J. Nuc1. Mat. 69 \& 70 (1978) 38.

/4/ Eyring, H., J. Chem. Phys. $\frac{3}{3}(1 \overline{935})$ 107, 492. Glasstone, S., Laidler, K.J. and Eyring, H., The Theory of Rate Processes, MC Graw Hill, New York, 1941.

/5/ Vineyard, G.H., J. Phys. Chem. Sol. 3 (1957) 121. Vineyard, G.H., and Krumhansl, Phys. Rev. 토 31 (1985) 4929.

/6/ Glyde, H.R., Rev. Mod. Phys. 39 (1967) 373.

17/ Delorenzi, G., Flynn, C.P. and Jacucci, G., Phys. Rev. B30 (1984) 5430.

/8/ Jacucci, G., Toller, M., Delorenzi, G. and Flynn, C.P., Phys. Rev. Lett. 52 (1984) 295, and to appear in Phys. Rev. B.

19/ Franklin, M.W., J. Chem. Phys. 52 (1942) 2659; in: Diffusion in Solids, A.S. Nowick and J.J. Burton, eds. (Academic Press, 1975) p. 1.

110/ Seeger, A. and Mehrer, H., in: Vacancies and Interstitials in Metals, North Holland, Amstexdam, 1970, p. 1; and Ref. /3/.

/11/ Peterson, N.I., Comments Solid state Phys. 8 (1978) 107.

/12/ Schilling, W., J. Nucl. Mat. $69 \& 70(1978)^{-465 .}$

113/ Carmeli, B. and Nitzan, A., Phys. Rev. A29 (1984) 1481; J. Chem. Phys. 80 (1984) 3596.

/14/ Kramers, H.A., Physica (Utrecht) 7 (1940) 284.

/15/ Hanggi, P., J. Stat. Phys., to appear in 1985.

/16/ Wolynes, P.G., Phys. Rev. Lett. 47 (1981) 968.

/17/ Grabert, H. and Weiss, U., Phys. Rev. Lett. $\frac{53}{73}$ (1984) 1787.

/18/ Grote, R.F. and Hynes, J.T., J. Chem. Phys. 73 (1980) $2715 ; 74$ (1981) 4465.

/19/ Skinner, J.L. and Wolynes, P.G., J. Chem. Phy̆s. 69 (1978) $21 \overline{43} ; \underline{72}$ (1980) 4913.

/20/ Büttiker, M., Harris, E.P. and Landauer, R., Phys. Rev. B28 (1983) 1268.

121/ Maradudin, A.A., Solid State Physics Vol. 19 (F. Seitz and D. Turnball, eds.), (1966) P. 51 .

122/ Klein, M.L., Barkex, J.A. and Koehler, T.R., Phys. Rev. B4 (1971) 1983

/23/ Stedman, R., Almqvist, L., Nilsson, G. and Raunio, G., Phys. Rev. 162 (1967) 545; Stedman, R. and Nilsson, G., Phys. Rev. 145 (1966) 492.

124/ Borkovec, M. and Berne, B.J., J. Chem. Phys. $\overline{82}$ (1985) 794 .

/25/ Zawadzki, G. and Hynes, J.T., Chem. Phys. Lett. 113 (1985) 476. 